\title{
Equilibrium and Kinetic Studies for The Adsorptive Removal of Lead (II) Ions from Aqueous Solution Using Activated Plantain Peel Biochar
}

\section{sciendo}

\author{
Nworie FS ${ }^{a^{*}}$, Oroke EC ${ }^{b}$, Ikelle IIc $\&$ Nworu JS \\ aDepartment of Industrial Chemistry, Ebonyi State University, P.M.B. 053 Abakaliki- Nigeria \\ bDepartment of Science Technology, The Federal Polytechnic, P.M.B. 5351 Ado-Ekiti-Nigeria \\ cDepartment of Chemistry, Nigeria Maritime University, Okerenkoko, Delta State, Nigeria \\ *Corresponding Author e-mail: nworie.sunday@ebsu.edu.ng
}

\section{Doi: 10.2478/acmy-2020-0002}

\section{Abstract:}

Studies on the adsorption of Pb(II) on plantain peels biochar (PPB) was conducted. The carbonized and activated, biochar was characterized using Braunauer-Emmett-Teller (BET) surface area and x-ray diffraction crystallography (XRD). BET analysis of the PPB indicated that the pore size (cc/g) and pore surface area $\left(\mathrm{m}^{2} / \mathrm{g}\right)$ was 8.79 and 16.69 respectively. Result of the XRD evaluated through Debye-Scherrer equation, showed a nanostructure with crystallite size of $14.56 \mathrm{~nm}$. Effects of initial metal ion concentration, $\mathrm{pH}$, and contact time were studied in a batch reaction process. Results showed that the adsorption of lead from aqueous solution increased with an increase in $\mathrm{pH}$ and initial concentration. Equilibrium modeling studies suggested that the data fitted mainly to the Langmuir isotherm. Adsorption kinetic data tested using various kinetic models fitted the Weber and Morris intraparticle diffusion model implicating pore diffusion as the main rate limiting step. The sorption studies indicated the potential of plantain peel biochar as an effective, efficient and low cost adsorbent for remediating lead (II) ions contaminated environment.

Keywords: Biochar, Adsorption, lead (II) ions, equilibrium model, kinetic model, BET

\subsection{Introduction:}

The indiscriminate discharge of heavy metals such as zinc, lead, cadmium through anthropogenic means into the environment has been reported as the major cause of water and soil pollution [1-3]. The presence of heavy metals on the environment is of global concern due to possible adverse effects on human health, as well as aquatic flora and fauna. A direct or secondary disposal heavy metal such as lead to soils and waters poses significant environmental risks, as they are non-degraded and, in high concentrations, are also very toxic [4]. It has been implicated among the most common global pollutants. This increasing rise in the concentration of lead on the environment is as a result of increasing population and anthropogenic activities in the developing nations. Its assimilation in relatively small amounts over a long period of time in the human body can lead to the malfunctioning of the organs and chronic toxicity. It has also been implicated as a major cause of miscarriages and deaths often experienced in mineral rich areas [5]. Lead is commonly present in effluents due to its wide industrial applications such as in mining, refineries, painting, metal plating and cleaning, battery and car radiator manufacturing and agricultural activities [6]. The removal of this toxic heavy metal is no doubt a worthwhile venture that should draw the attention of researchers and relevant government agencies.

Biochar is a carbon rich, solid by-product resulting from the pyrolysis of biomass under oxygen-free and low temperature conditions. Biochar's proven ability to remain stable against chemical and biological degradation, when applied to soils, makes it a pioneer means of mitigating climate change [4]. Biochar from plantain peels have been proven to exert effects on clinically important agents and are important as good adsorbent [7]. The indiscriminate disposal of plantain peels constitute serious environmental hazard as it oftentimes leads to clogging of waterways causing flooding and also converting the town centers to foul smelling scenes. Peels of plantain are wastes and are shown to be a very good source of phyto-chemicals and dietary fibers which are beneficial to human body [7]. Converting plantain peel waste into biochar for lead adsorption is a green approach for waste re-utilization and biochar has long been used as a sequestering agent for heavy metals, dyes and other environmental contaminants.

Various techniques are available for the removal heavy metals from wastewaters, surface and underground waters. These methods are chemical precipitation, ion exchange, filtration, evaporation, reverse osmosis, solvent extraction, electrochemical treatment, membrane separation and activated carbon adsorption [7-9]. However, all these methods are either inefficient or not economical when heavy metal exists in lower concentrations [8]. For instance, activated carbon a commonly used adsorbent for removal of various pollutants from wastewater has been jettisoned due to its high cost [9].

Consequently, it is important to find new methods for removing heavy metals from water and wastewater. Comparing with other conventional treatment techniques, adsorption was found to be a promising alternative technique for the removal of trace amount of heavy metals from water and wastewater. This is due to its convenience, ease of operation, and versatility. It minimizes or removes different types of pollutants, thus giving it a wide range of applications in wastewater treatment [9]. Predicting the rate at which adsorption takes place for a given system among other things, is the most important factor in adsorption system design, with adsorbate residence time and the reactor dimensions controlled by the system's kinetics [1]. Adsorption is a surface phenomenon that occurs when a solute is preferentially retained on the surface of an adsorbent forming a thin layer of the adsorbate [2].

The benefit or objectives of this research is bipodal as waste biomass of plantain peel which constitute serious environmental menance was converted to an environmentally friendly material for sustainable development. This involves the conversion of plantain peel to biochar by pyrolysis, activation of the biochar for surface functionality and secondly, lead (II) ions which has remained a problem for flora and fauna in water bodies as well as in other environmental matrices are removed by batch adsorption process. The adsorption of lead from aqueous was modeled using adsorption isotherms and kinetic models to identify the sorption mechanism. The batch adsorption process was studied under different conditions of time of contact, initial metal ion concentration and $\mathrm{pH}$. The work is a clean- up exercise aimed at removing contaminants from water bodies. It is a continuation of our former work on the removal of zinc (II) ions from water samples previously published [7]. The main objective of this study was to synthesize, activate and apply plantain peel biochar for the removal of lead and thereafter profile the equilibrium and kinetic studies using the simulated data.

\subsection{Materials and Methods}

\subsection{Materials}

Except otherwise stated, all analytical grade reagents used for the study ranging from lead acetate, $\mathrm{Pb}_{(\mathrm{CH}} \mathrm{COO}^{2+}, \mathrm{H}_{2} \mathrm{SO}_{4}, \mathrm{HNO}_{3}, \mathrm{Na}_{2} \mathrm{CO}_{3}, \mathrm{NaOH}_{2} \mathrm{Eriochrome}$ black-T, EDTA and $\mathrm{NaCl}$, were all obtained from Sigma Aldrich ${ }^{\circledR}$ and used without further purification. More so, to avoid interference of radicals, double distilled water was used for all experiments.

\subsection{Characterization of the activated plantain peel biochar}

The BET surface area was measured using Micromeritics ASAP 2020 system. X-ray diffraction pattern was obtained on a Bruker® D8 Discover diffractometer, equipped with a Lynx Eye detector, under $\mathrm{Cu}$-Ka radiation $(\mathrm{l} 1 / 41.5405 \AA)$. With data collected in the range of $2 \theta=10$ to $100^{\circ}$, scanning rate at $0.010^{\circ} \mathrm{min}^{-1}, 192 \mathrm{~s}$ per step and samples placed on a zero background silicon wafer slide.

\subsection{Sample Collection and Preparation}

The unripe plantain (Musa paradisiaca) was harvested from Abakaliki metropolis in Ebonyi state of Nigeria in a sac bag. The peels were carefully removed cleaned of soil and other impurities, sun dried to reduce moisture content for 7 days and then oven dried at $60 \pm 1^{\circ} \mathrm{C}$ for $24 \mathrm{~h}$. The modified method $(13)$ was adopted for carbonization of the plantain peels. This was done at a temperature of $600 \pm 1{ }^{\circ} \mathrm{C}$ in nitrogen environment in a muffle furnace for $6 \mathrm{~h}$. The 
resulting biochar was cooled at room temperature, ground to fine powder, and then subjected to activation. A portion of $10 \mathrm{~g}$ of the carbonized biochar was measured into a $500 \mathrm{~mL}$ beaker and $30 \mathrm{~mL}$ of concentrated $\mathrm{HNO}_{3}$ added with stirring for $2 \mathrm{~h}$ continuously. The mixture was diluted with deionized water and decanted several times and then washed with double distilled water until the $\mathrm{pH}$ was tested to be 6.5. The activated plantain peel biochar (APPB) was then dried in an oven at a temperature of $110 \pm 1^{\circ} \mathrm{C}$.

2.4. Adsorption of $\mathrm{Pb}(\mathrm{II})$ ions by batch process

Batch experiments were done to obtain adsorption data by varying different conditions of contact time, $\mathrm{pH}$ and initial metal ion concentration. The adsorption of $\mathrm{Pb}(\mathrm{II})$ ions from aqueous solution was investigated by initially adding $0.1 \mathrm{~g}$ of the adsorbent to $10 \mathrm{~mL} 0.01 \mathrm{M} \mathrm{Pb}$ (II) ions aqueous solution in a $500 \mathrm{~mL}$ vessel at room temperature $\left(30 \pm 1{ }^{\circ} \mathrm{C}\right)$ for $150 \mathrm{~min}$. The solution was equilibrated using mechanical shaker and resulting solution filtered. The isotherm and kinetics experiments enabled elucidation of the characteristics and mechanism of the adsorption of lead onto the activated plantain peel biochar. For each experiment, $0.1 \mathrm{~g}$ of the activated biochar was mixed with $10 \mathrm{~mL}$ lead solution in the vessel. The mixture was shaken at $100 \mathrm{rpm}$ on a rotary mechanical shaker at room temperature.

Consequently to measure the sorption kinetics, $0.01 \mathrm{M}$ lead solution was used while varying time between 30-150 min. To get sorption isotherms, $0.1 \mathrm{~g}$ of the activated biochar was added to different concentrations of lead solution $(4,8,12,16$ and $20 \mathrm{mg} / \mathrm{ml})$ and the mixtures equilibrated for 120 seconds each Similar method was applied for $\mathrm{pH}$ varied between 2-10. The mixtures were separated by filtration using Whatman No1 filter paper at the end of each experiment. Equation 1 was used to evaluate concentration of lead in the filtrate.

Concentration of $\mathrm{Pb}(\mathrm{II})$ ion $=\frac{\text { Molarity of EDTA } \times \text { Volume of EDTA }}{\text { Volume of } \mathrm{Pb}(\mathrm{II}) \text { ion solution }}$

Equation 2 shows that the mole ratio of combination of EDTA with Pb(II) ion is 1:1

$\mathrm{Pb}^{2+}+\mathrm{EDTA}^{4-} \longrightarrow \mathrm{Pb}[\mathrm{EDTA}]^{2+}$

The amount of $\mathrm{Pb}(\mathrm{II})$ ion adsorbed per unit mass of adsorbent at equilibrium, (qe) (mg/g) was evaluated using the relation in Equation 3.

$\mathrm{qe}=\frac{C o}{C e} X \frac{V}{M}$

Where $\mathrm{Co}$ is initial concentration of $\mathrm{Pb}(\mathrm{II})$ ions in solution, $\mathrm{Ce}$ is the equilibrium concentration of $\mathrm{Pb}(\mathrm{II})$ ions, $\mathrm{V}$ is the volume of the solution and $\mathrm{M}$ the mass of the activated plantain peel adsorbent

\subsection{Results and Discussion}

\subsection{Brunauer, Emmett, Teller (BET) surface area characterization}

BET surface area was applied to determine the surface area and pore size of the plantain peel biochar. The pore size $(\mathrm{cc} / \mathrm{g})$ and pore surface area $(\mathrm{m} 2 / \mathrm{g})$ of the biochar was 8.79 and 16.69 respectively and represented in Figure 1. The large surface area per gram of a sample of the biochar indicates that there is less erosion and more ability to capture metallic particulates present in a given media [2]. BET surface area analysis and XRD analysis of the biochar showed nanosize forms of the biochar typical of a nanocrystalline material.

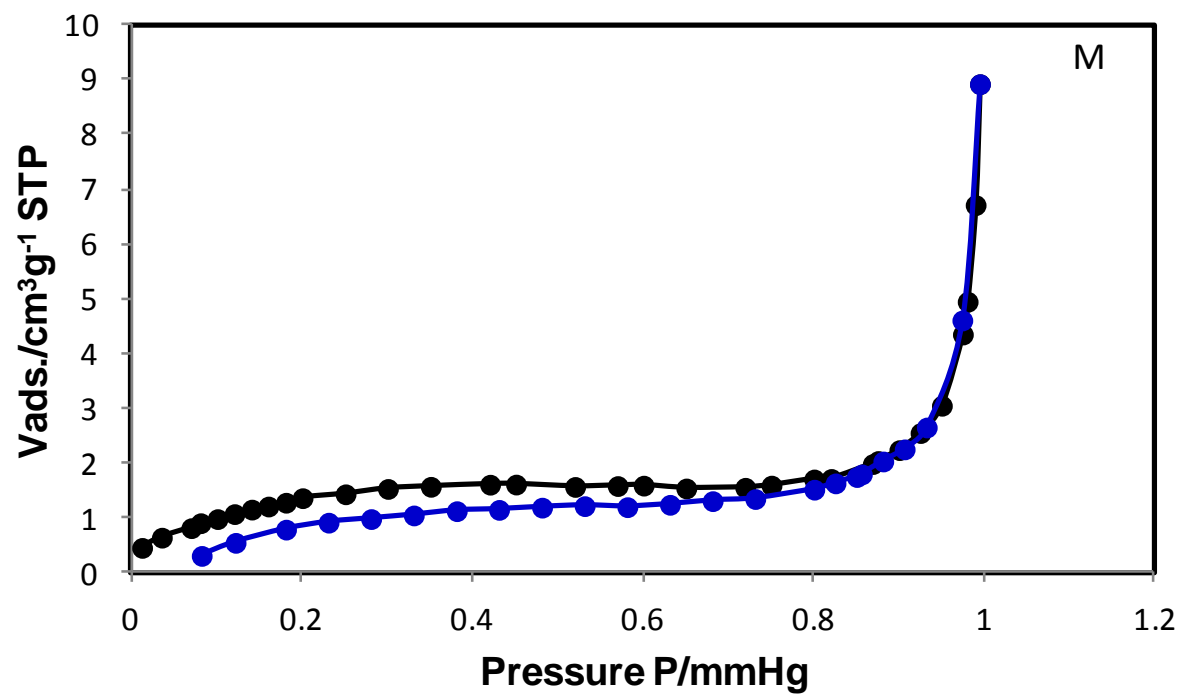

Figure 1: BET surface area plot of activated plantain peel biochar

\subsection{XRD analysis of the solid sorbent}

The x-ray diffraction pattern of the activated plantain peel biochar (Figure 2) indicated a crystalline nature with significant intense peak observed at $2 \theta=$ 40.65 with observed decomposition of some compound after forming the biochar of plantain peels [7]. The crystallite size (nm) was calculated from DebyeScherrer equation (Equation 4):

$d=\frac{\mathrm{K} \lambda}{\beta \operatorname{Cos} \theta}$

Where $\mathrm{K}$ is Debye-Scherer constant, $\beta$ is the full width at half maximum, is the wavelength and $\theta$ is Bragg angle. The average crystal size of the particles as calculated from the width of the XRD peaks using Debye-Scherrer equation was $14.56 \mathrm{~nm}$ indicating that the biochar is nano-sized (17). 


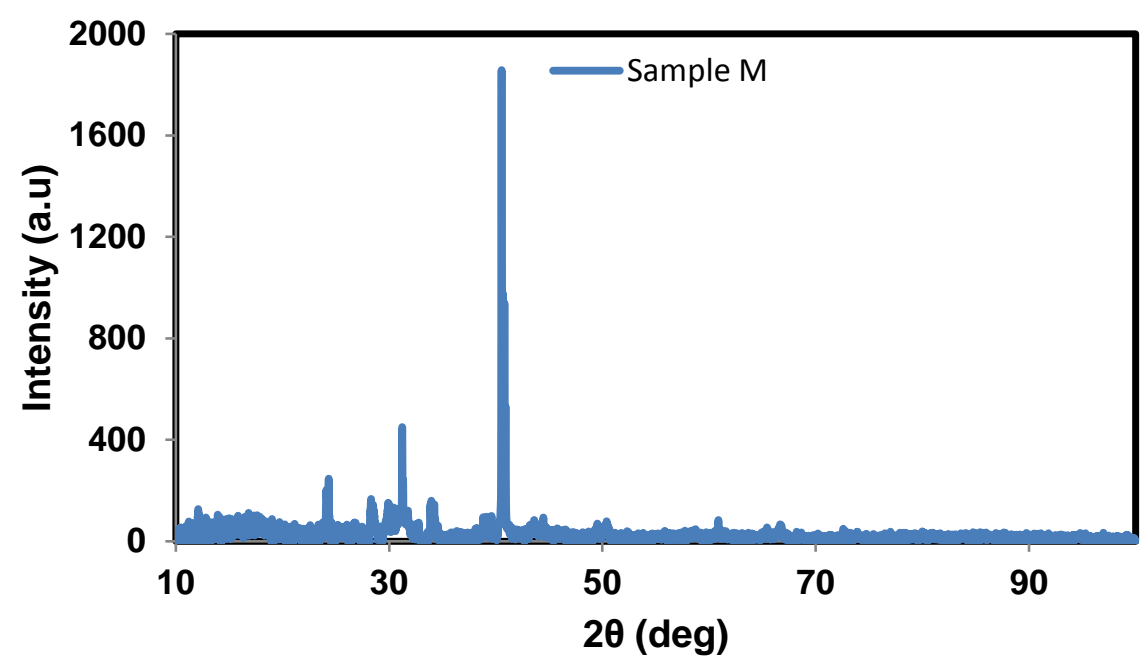

Figure 2: X-ray diffractogram of the activated plantain peel biochar

\subsection{Effect of $\mathrm{pH}$ on the adsorption of $\mathrm{Pb}^{2+}$ on the APPB}

Batch adsorption process solution $\mathrm{pH}$ is one of the most important parameters which influences the surface charge and dissociation of functional groups on the adsorbent $[7,10]$. The adsorption capacities of lead onto plantain peel biochar were strongly affected by the $\mathrm{pH}$ (Figure 3). There was a corresponding increase in the equilibrium adsorption of $\mathrm{Pb}$ (II) ion as the $\mathrm{pH}$ was varied from 2 - 10, with $\mathrm{pH} 10$ having the highest adsorption capacity. From the result, it could be because at low $\mathrm{pH}$, there was an inhibition of the removal of $\mathrm{Pb}(\mathrm{II})$ ion because the competition of $\mathrm{H}+$ and $\mathrm{Pb}$ (II) for adsorption on the active sites. Hence it was difficult for the metal ions to move toward the positively charged surface of PPB due to electrostatic repulsion and hence a lower removal was observed at low $\mathrm{pH}$ values [6].

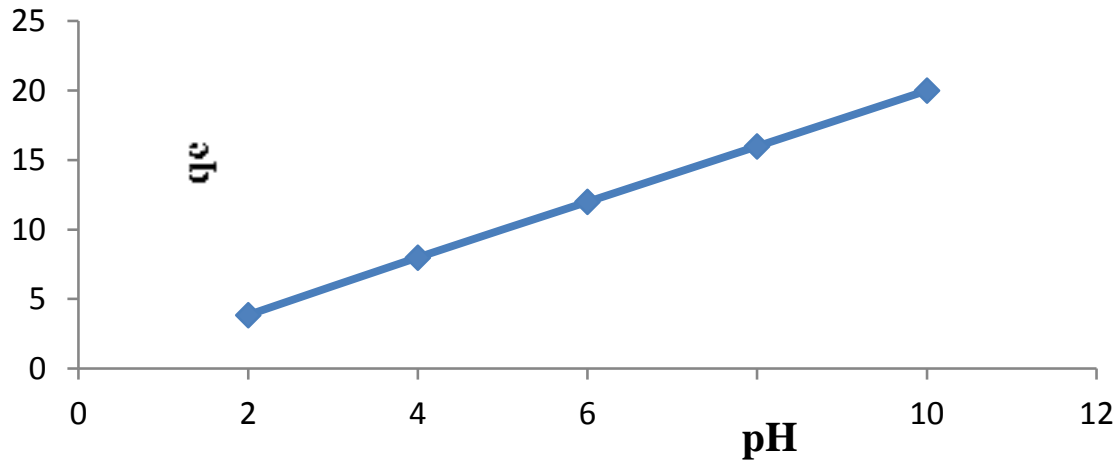

Figure 3: Effect of $\mathrm{pH}$ on adsorption equilibrium capacity of activated $\mathrm{PPB}$ at $30 \pm 1^{\circ} \mathrm{C}$

\subsection{Effect of time of contact and initial metal ion concentration}

Contact time is the time required for the system to reach equilibrium. This solid/liquid heterogeneous system frequently undergoes different mass transfer steps, some of which could be comparatively slow. Hence, it is necessary to determine the contact time so as to ensure that the equilibrium is reached [9]. Adsorption of $\mathrm{Pb}(\mathrm{II})$ ions was observed to increase with increased contact time (Figure 4) until equilibrium was attained. This could be that as adsorption starts, the active sites available got occupied and are co-ordinatively consumed with time thereby clogging the sorption sites hence leading to the reduction or unavailability of free site [7]. Variation of the initial metal ion concentration from 4, 8, 12, 16 and $20 \mathrm{mg} / \mathrm{ml}$ showed gradual increase in the percentage of metal adsorbed until equilibrium was reached at initial metal ion concentration of $16 \mathrm{mg} / \mathrm{ml}$. Further increase in the concentration of the metal ion decreases the quantity adsorbed at $\mathrm{pH} 10$ at room temperature. The decrease could be that the adsorptive sites are saturated until no more exposed surface resulting from intermolecular hydrogen bonding and hence regress in the amount adsorbed [3].

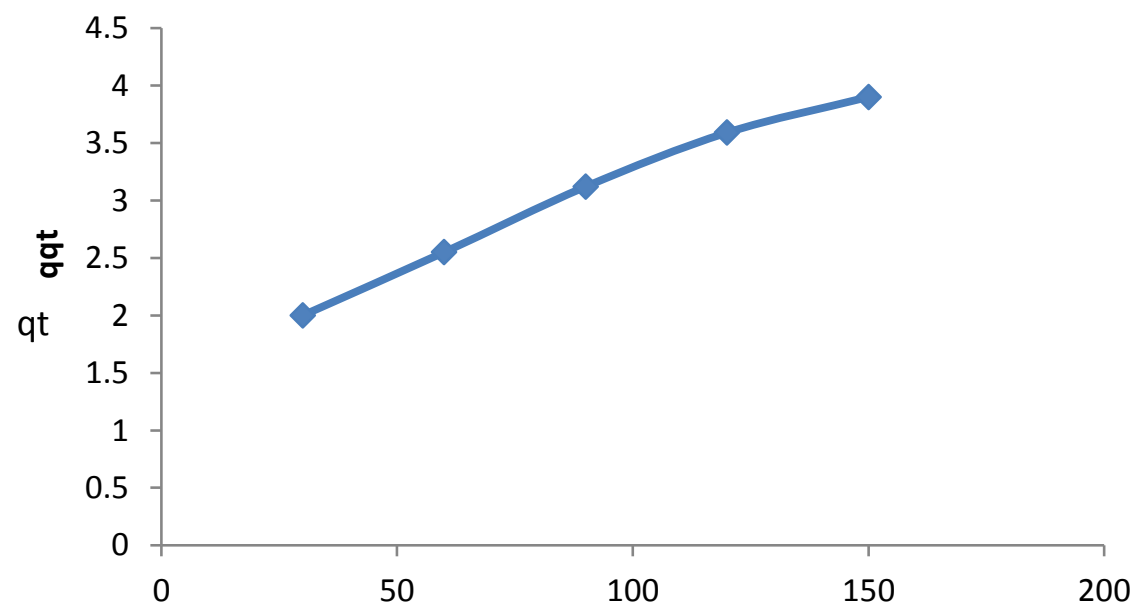

$\mathbf{t}(\min )$

Figure 4: Effect of contact time on the adsorption of $\mathrm{Pb}^{2+}$ on Plantain peels biochar at $\mathrm{pH} 10$ and at $30 \pm 1^{\circ} \mathrm{C}$ 


\subsection{Langmuir isotherm}

The Langmuir adsorption isotherm has been used to predict the performance of different biosorbents. It assumes that adsorption takes place on homogenous site and ceases as sites become unavailable. The adsorption is thus monolayer without established interaction between the adsorbate [7, 11, 12]. Langmuir model is expressed with Equation 4.

$\mathrm{qe}=\mathrm{Qm} \frac{\mathrm{bCe}}{1}+\mathrm{bCe}$

$\mathrm{Ce}(\mathrm{mg} / \mathrm{g})$ is the concentration of adsorbate at equilibrium, $\mathrm{qe}(\mathrm{mg} / \mathrm{g})$ the mass of adsorbate, $\mathrm{Qm}$ and $\mathrm{b}$ are constants regarded as Langmuir constants and stands for maximum adsorption capacity and adsorptive bonding energy respectively determined graphically by linearizing Langmuir isotherm as shown in Equation 4a.

$\frac{C e}{q e}=\frac{\mathrm{Ce}}{Q m}+\frac{1}{b Q m}$

$\mathrm{Qm}$ and $\mathrm{b}$ are constants from the graphical plot of $\frac{C e}{q e}$ against $C e$ and represented as slope and intercept. From our result (Figure 5), the correlation coefficient $\left(\mathrm{R}^{2}\right)$ is 0.998 indicating a perfect fit of the monolayer adsorption [2]. It also shows that the adsorption sites are energetically identical and equivalent, structurally homogeneous and no observed interaction between the molecules adsorbed and close sites. Transfer of the adsorbate from the available sites to another is inhibited in the surface of the sites and the plantain peel biochar has finite adsorptive capacity for the $\mathrm{Pb}(\mathrm{II})$ ions.

However, to evaluate the dimensionless separation factor a measure of the desirability and favourability of the model, the Langmuir parameter b regarded as Langmuir isotherm model constant was employed and values optimized from Hall equation $[7,13]$ (Equation 4b)

$R L=\frac{1}{1+\mathrm{bCo}}$

Where $\mathrm{Co}$ is the initial concentration of $\mathrm{Pb}(\mathrm{II})$ ion and the values of $\mathrm{b}$ was found to be between 0 and I indicating the favorability of the adsorption process [7]

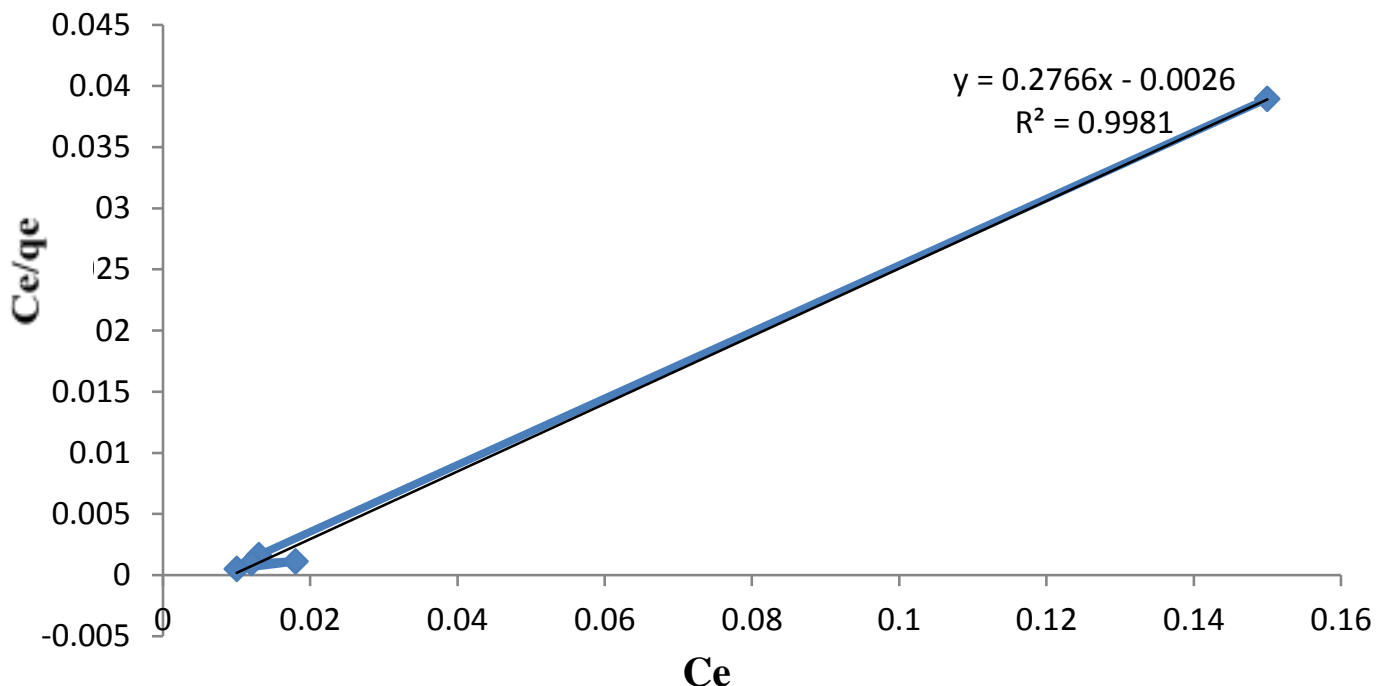

Figure 5: Linear plot of Langmuir isotherm of $\mathrm{Pb}(\mathrm{II})$ ion on Plantain peels biochar

\subsection{Freundlich Isotherm}

Freundlich sorption isotherm model considers the adsorption to occur in a heterogeneous surface where there is no limited degree of adsorption with sites and energies exponentially distributed. It describes reversible adsorption and is not restricted to the formation of monolayer. Equation 5 is an expression for Freundlich sorption isotherm. Where $\mathrm{n}$ is the adsorption intensity, $\mathrm{K}_{\mathrm{F}}$ is the adsorption capacity of the biochar obtained as the intercept and slope respectively and shown as the linear plot of Inqe against InCe illustrated in Equation 6. The value of correlation coefficient was 0.717 (Figure 7), indicating that the adsorption is best fitted to Langmuir isotherm than does Freundlich isotherm. $\mathrm{qe}=K_{F} C e^{1 / n}$

Inqe $=\operatorname{InK} \mathrm{F}_{\mathrm{F}}+\frac{1}{n} \operatorname{InC} e$

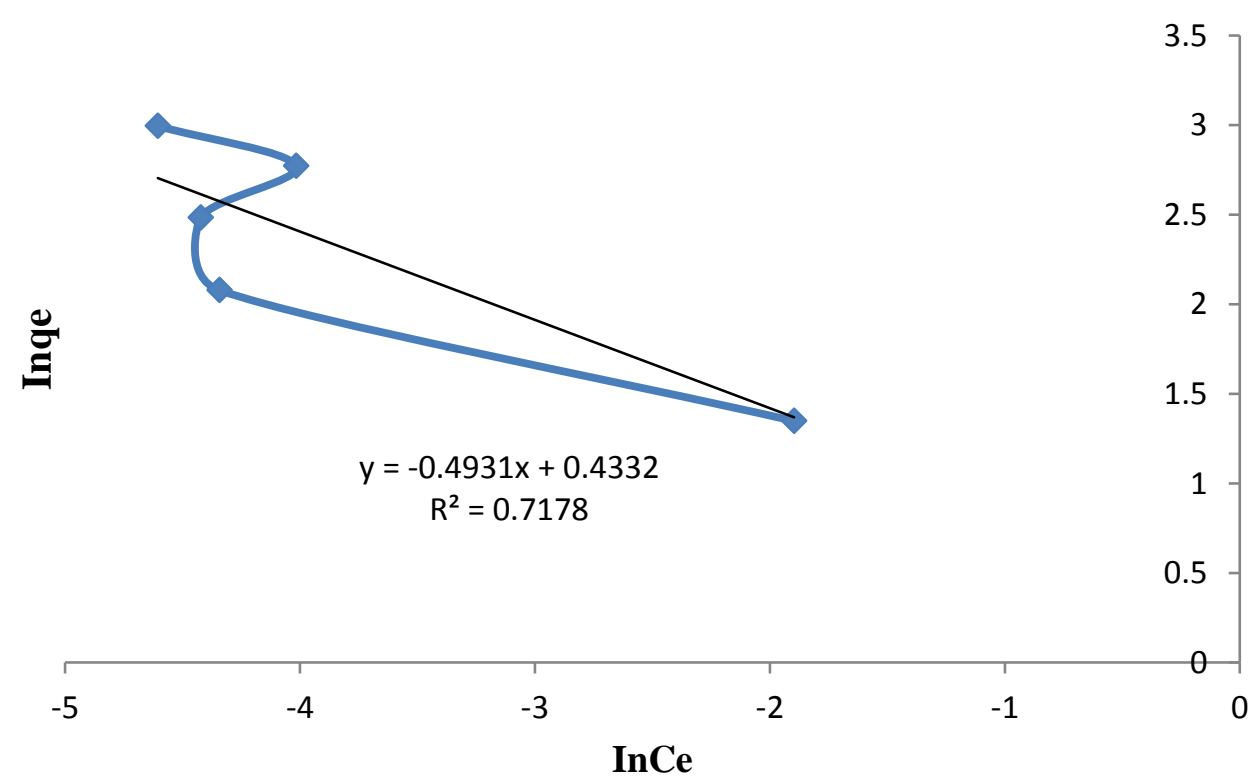

Figure 6: Freundlich adsorption isotherm for the adsorption of $\mathrm{Pb}(\mathrm{II})$ ions onto activated plantain peel biochar. 


\subsection{The Jovanovic isotherm}

Jovanovic isotherm model also assumes a close approximation for monolayer localized adsorption without lateral interactions. It is similar to Langmuir model, except that the Jovanovic model allows surface binding vibrations of an adsorbed species [3,12]. The linear form of the model is shown in equation 7 and a correlation coefficient $\left(\mathrm{R}^{2}\right)$ of 0.722 obtained from the plot of Inqe against Ce (Figure 7). This shows that the simulated data did not obey this model.

$\operatorname{Inq}_{\mathrm{e}}=\operatorname{Inq} \mathrm{q}_{\mathrm{m}}-\mathrm{K}_{\mathrm{f}} \mathrm{C}_{\mathrm{e}}$

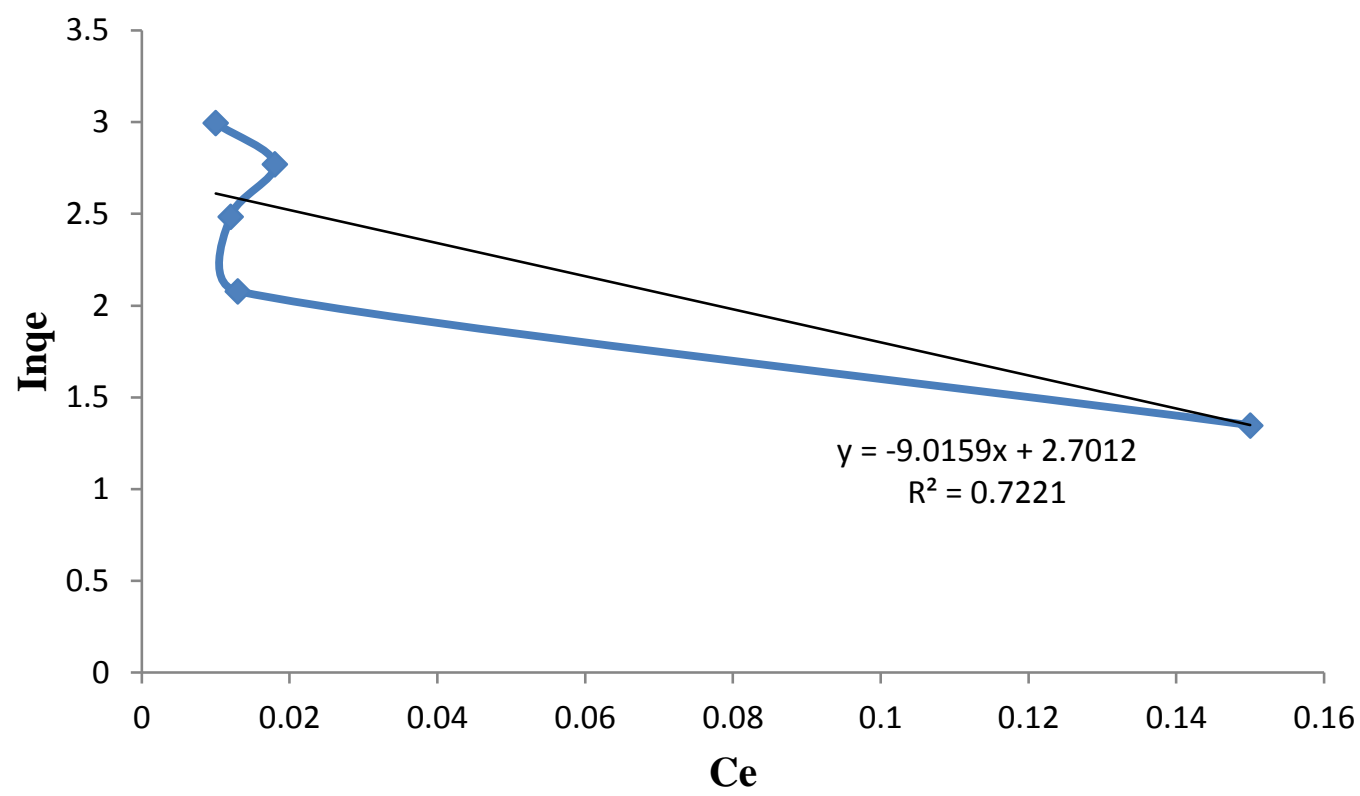

Figure 7: Jovanovich adsorption Isotherm for the adsorption of zinc(II) ions onto activated plantain peel biochar.

\subsection{Temkin isotherm}

This model is often applied under the condition of intermediate mode of sorbate concentration. It assumes that the fall in the heat of sorption of molecules in the layer to decreases linearly with area of coverage owing to sorbent or sorbate interaction instead of logarithmic (Nworie, 2019). Equation 8 is the linear form of Temkin isotherm model.

$\mathrm{q} e=\beta \operatorname{In} A_{t}+\beta \operatorname{InC} e$

Where $\beta$ is related to the heat of sorption, $A$ is the binding constant at equilibrium state. The plot of qe against InCe gives the slope and intercept as $A_{t}$ and $\beta$ respectively. Figure 8 showed the value of $\mathrm{R}^{2}$ as 0.526 , which is very much lesser than Langmuir, Freundlich and Jovanovich isotherms respectively. The implication of this is that the adsorption does not follow the Temkin model.

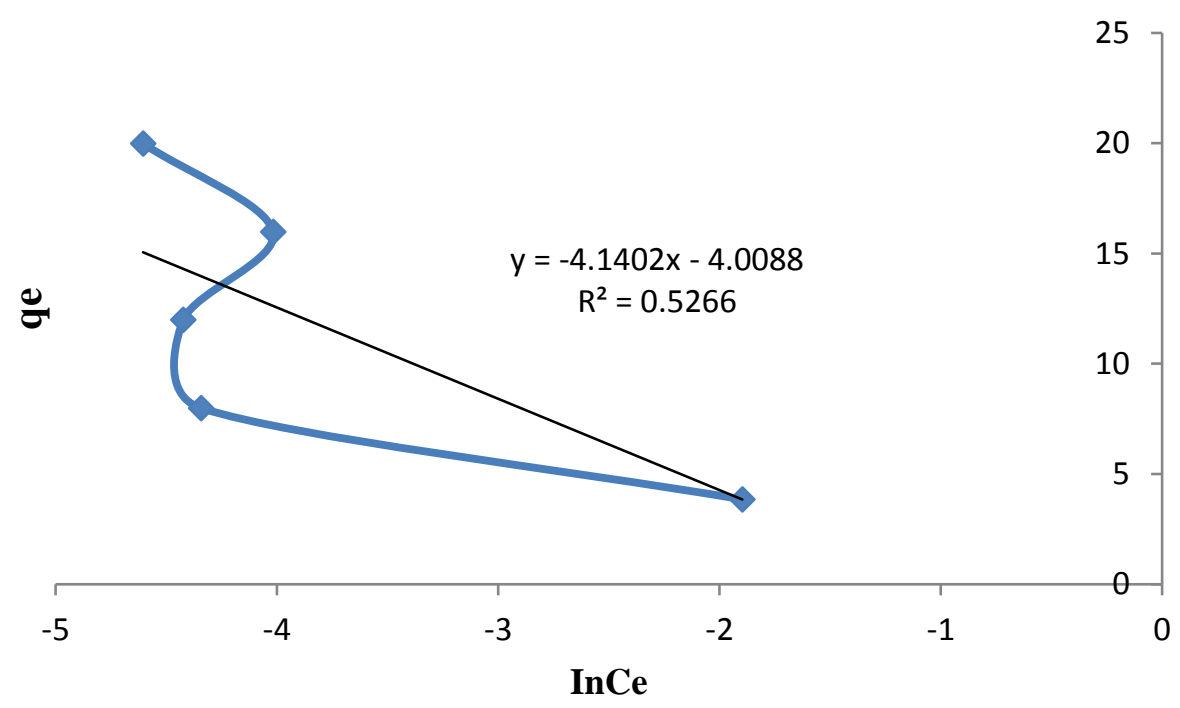

Figure 8: Temkin adsorption isotherm for the adsorption of $\mathrm{Pb}(\mathrm{II})$ ions onto activated plantain peel biochar

\subsection{Elovich sorption isotherm}

Elovich model assumes that the actual adsorbent surfaces are energetically heterogeneous. It is based on multilayer adsorption and adsorption increases exponentially with adsorption site. The equation defining the model is based on a kinetic principle which assumes that the adsorption sites increase exponentially with adsorption, which implying a multilayer adsorption [12]. A plot of In(qe/Ce) versus qe shows the adsorption regression coefficient as 0.717 , which goes a long way to show that the adsorption was on a monolayer.

$\frac{q e}{q m}=\mathrm{KECE} e^{\frac{q e}{q m}}$ 


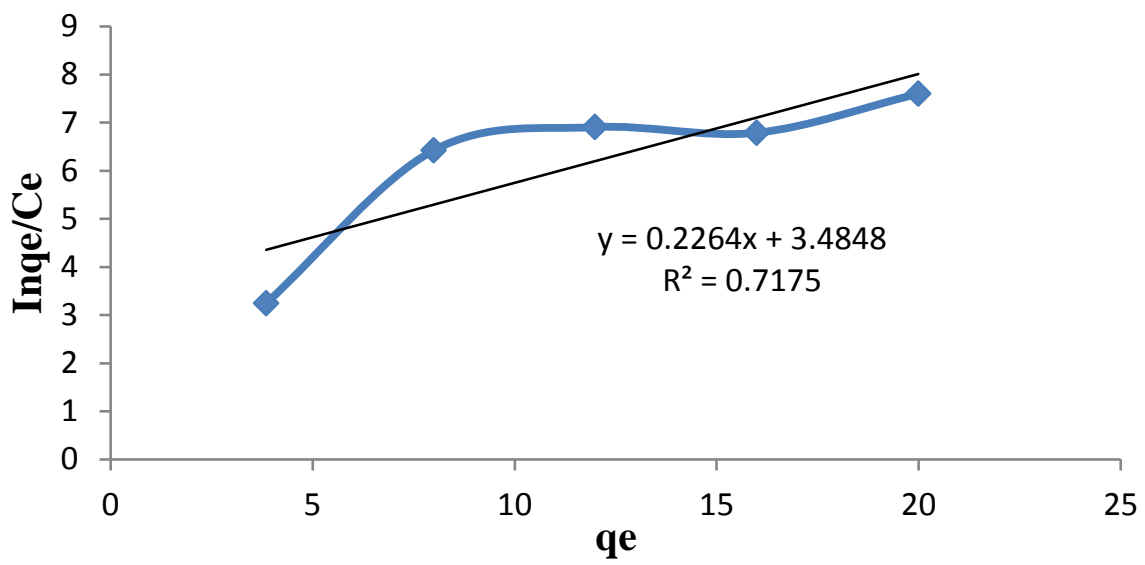

Figure 9: Elovich sorption isotherm for the adsorption of zinc(II) ions onto activated plantain peel biochar

\subsection{The Boyd Kinetics Model}

In a bid to determine the actual rate-determining step involved in the adsorption processes and to evaluate the slow step involved in the adsorption process [14], the kinetics data were also subjected to Boyd kinetic model of analysis (Equation 10).

$\mathrm{Bt}=-0.4977-\ln (1-\mathrm{F})$

where $\mathrm{F}$ represents the fraction of solute adsorbed at any time, $\mathrm{t}(\mathrm{min})$, calculated from $\mathrm{F}=\mathrm{qt} / \mathrm{qo}$. Hence,

$\mathrm{Bt}=-0.4978-\ln (1-\mathrm{qt} / \mathrm{qo})$

The plots of Bt against time (minutes) as shown in Figure 10 are not straight lines, and do not pass through the origin therefore, we can conclude that the adsorption mechanism is mainly governed by film diffusion.

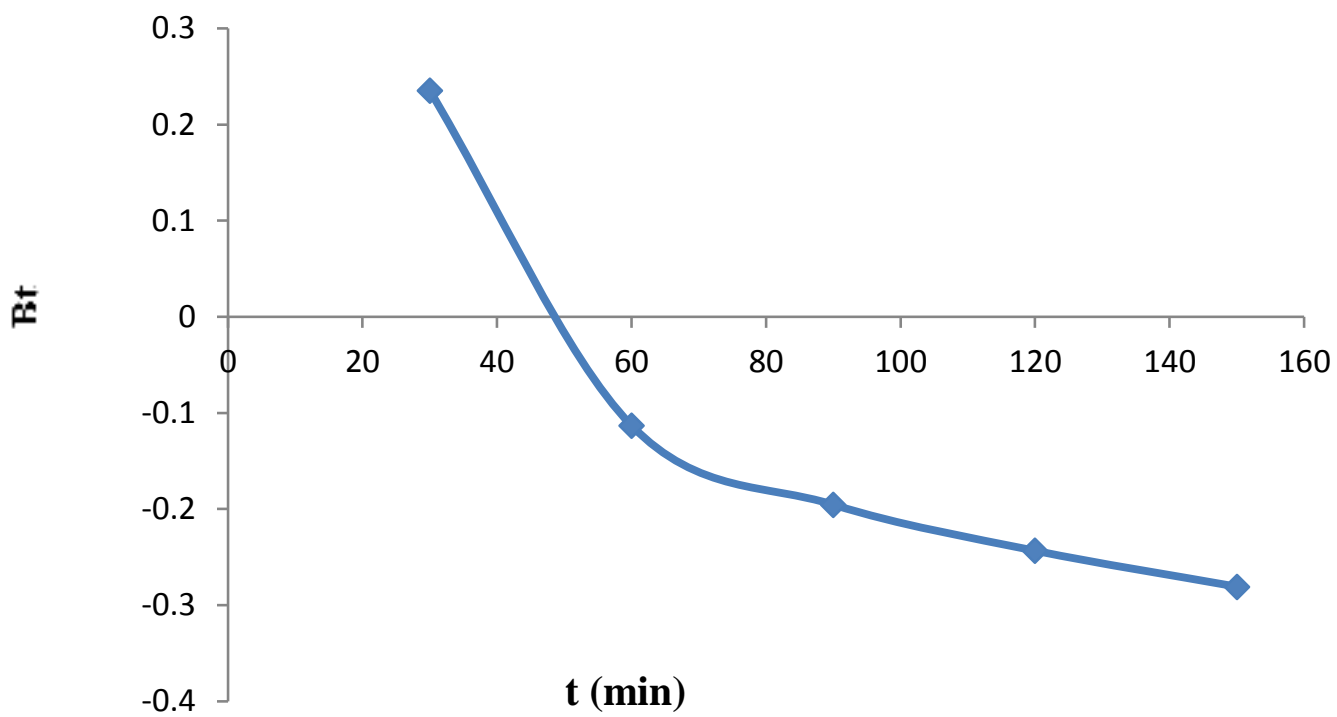

Figure 10: Boyd kinetic plot for adsorption of $\mathrm{Pb}^{2+}$ on Plantain peels biochar at $298 \mathrm{~K}$

\subsection{Weber and Morris Intra-particle diffusion model}

The intra-particle diffusion model was plotted in order to verify the influence of mass transfer resistance on the binding of $\mathrm{Pb}^{2+}$ on $\mathrm{Plantain}$ peels biochar The kinetic results were analysed by the Weber and Morris intraparticle diffusion model to elucidate the diffusion mechanism, the model is expressed in equation 11:

$\mathrm{qt}=\mathrm{k}_{\mathrm{p}} \mathrm{t}^{1 / 2}+\mathrm{C}$

11

where $C$ is the intercept and $k_{\mathrm{p}}$ is the intraparticle diffusion rate constant $(\mathrm{mg} / \mathrm{g} / \mathrm{min} 1 / 2)$, which can be evaluated from the slope in the linear plot of $q \mathrm{t}$ against $t^{1 / 2}$. The intercept of the plot reflects the boundary layer effect [3]. The larger is the intercept, the greater will be the contribution of the surface sorption in the rate-controlling step. If the regression in the plot of $q$ t versus $t^{1 / 2}$ is linear and passes through the origin, then intra-particle diffusion is the sole rate-limiting step. However, the linear plots at each concentration did not pass through the origin. This deviation from the origin may perhaps be due to the difference in the rate of mass transfer in the initial and final stages of adsorption. This indicated that there is some degree of boundary layer contro and this further showed that the intra-particle diffusion was not only the rate-limiting step, but also be the rate controlling of sorption or that all may be operating simultaneously [15]. This could also be seen on the high regression coefficient value (Figure 11). 


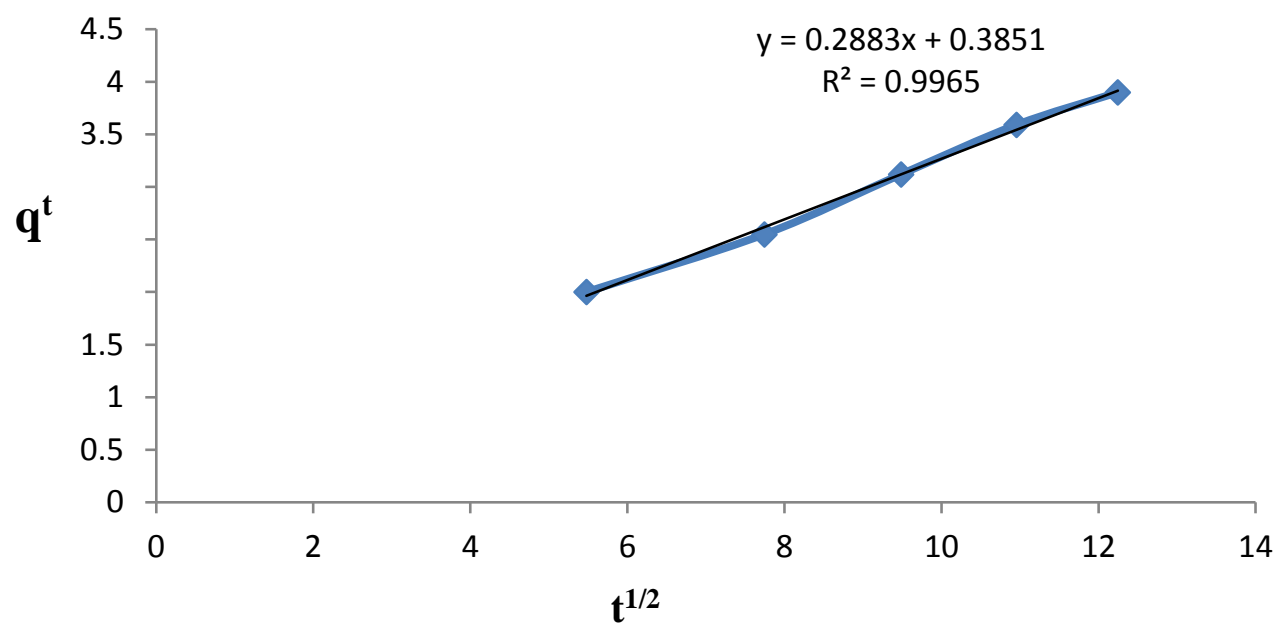

Figure 11: Plot for Weber and Morris intra-particle diffusion model

\subsection{Pseudo-first- order model}

The sorption of adsorbate from liquid phase is of great importance and remains the most widely used Lagergren's rate equation [7]. Equation 12 is the linearized pseudo-first order model with $\mathrm{t}$ as time of contact, qt as mass of $\mathrm{Pb}(\mathrm{II})$ ions, qe is equilibrium mass of $\mathrm{Pb}(\mathrm{II})$ ions adsorbed and $\mathrm{K}_{1}$, pseudo first order constant. From Figure 12, the data have high regression coefficient of 0.911but lower than the pseudo-second order model showing that the sorption process does not agree with the first order model.

$\operatorname{In}(\mathrm{qe}-\mathrm{qt})=\operatorname{In} q e-k_{1} t$

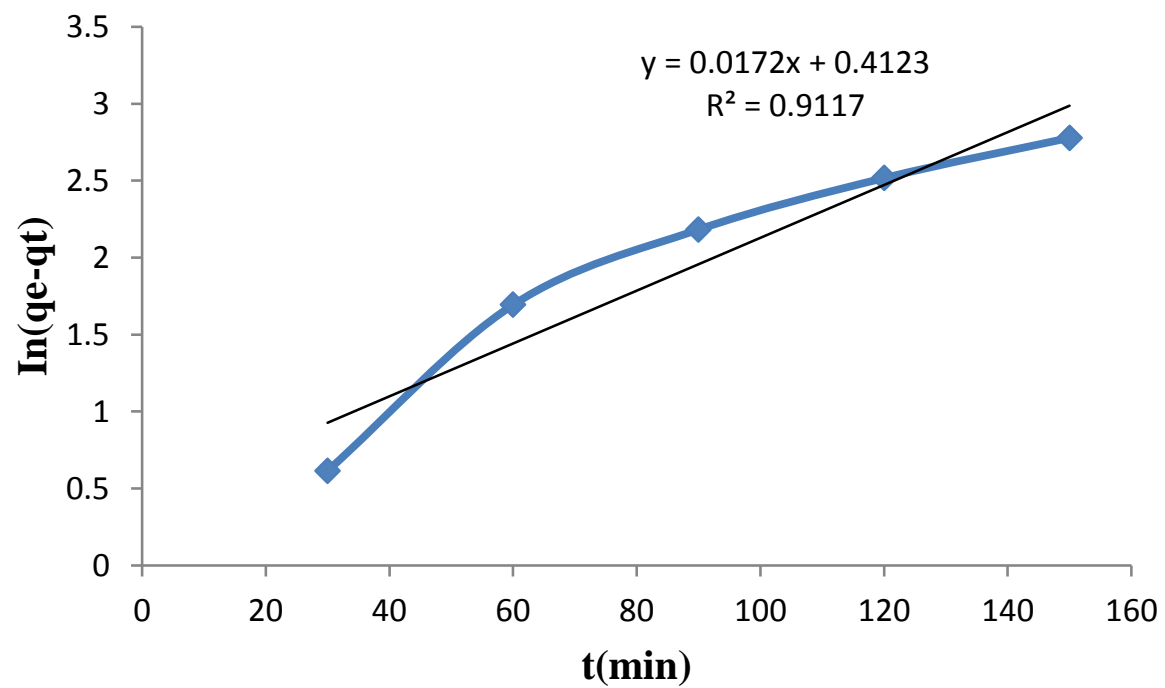

Figure 12: Pseudo-first-order kinetic plot for the adsorption of pb(II) ions onto activated plantain peel biochar.

3.13. Pseudo-second-order kinetic model

Chemisorption defines the rate-controlling step in pseudo-second-order process. Pseudo-second-order rate expression was used in the present study to describe chemisorption involving valency forces through the sharing or exchange of electrons between the adsorbent and adsorbate as covalent forces, and ion exchange [1] as illustrated in Equation 13 (12).

$t / q t=1 / K_{2}^{q e 2+t / q e}$

Where $K_{2}$ is pseudo second order constant. The regression coefficient $\left(R^{2}\right)$, of the obtained data as shown in Figure 13, was 0.982 ; indicates a good relationship between the parameters and shows that the adsorption kinetic model could be in control of the process.

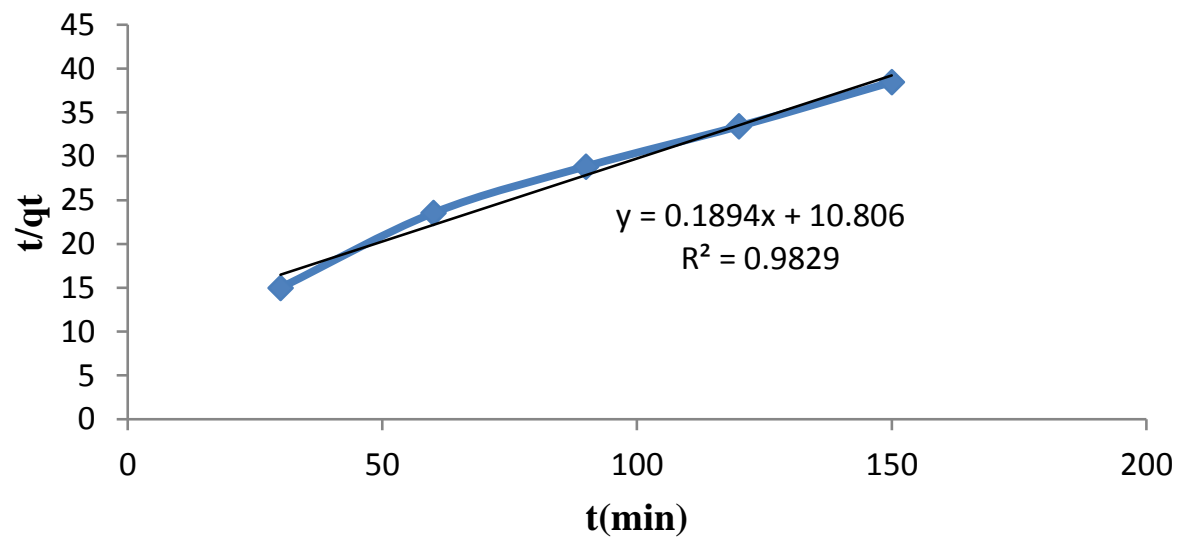


Figure 13: Pseudo-second-order kinetics plot for the adsorption of $\mathrm{Pb}(\mathrm{II})$ ions onto activated plantain peel biochar

\subsection{Conclusion}

The presence of $\mathrm{Pb}(\mathrm{II})$ ion from aqueous media was removed by adsorption using an effective, efficient and low cost plantain peel biochar. XRD analysis of the biochar indicated that it is of nanosize form corroborating the BET surface analysis of pore size and pore surface area typical of a nanocrystalline material. $\mathrm{Pb}(\mathrm{II})$ ions sorption was found to increase with increase in time until equilibrium at 120 minutes but decreased with increase in $\mathrm{pH}$ and initial $\mathrm{Pb}(\mathrm{II}) \mathrm{II})$ ion concentration. Sorption equilibrium and kinetic models were applied to test the effectiveness, efficiency and applicability of the processes. The adsorption isotherm obeyed Langmuir monolayer process whereas the kinetic mechanism showed mainly pore diffusion process been prevalent. The study serves as a green approach to the removal of $\mathrm{Pb}(\mathrm{II})$ (II) ions from contaminated media. The method is green because it sequestrates metal contaminants from the environment without contaminating the environment. A green or benign analytical approach is an environmentally friendly approach for which the adsorption process presents. The zinc metal can be eluted or desorbed from the activated plantain peel biochar for reuse or before discarding to the environment as a soil amender.

\section{References}

[1] S. Banerjee, M.C. Chattopadyaya. "Adsorption characteristics for the removal of a toxic dye, tartrazine from aqueous solutions by a low cost agricultural by-product", Arabian Journal of Chemistry, Vol. 10, Pp. S1629-S1638, 2015. Doi: https://doi.org/10.1016/ j.arabjc .2013.06.005

[2] L. Cui, C. Chen, C. Yen, I. Yan, J.A. Ippolito, Q. Hussain, “Mechanism of Adsorption of Cadmium and Lead by Iron-Activated Biochar”, Bioresources, Vol. 14, Pp. 842-857, 2020. Doi: 10.1016/j.biortech.2017.01.074

[3] F.S. Nworie, F.I. Nwabue, W. Oti, E. Mbam, B.U. Nwali. "Removal of Methylene Blue from Aqueous Solution using Activated Rice. Husk Biochar Adsorption Isotherms, Kinetics and Error Analysis", Journal of Chilean Chemical Society, Vol. 64, Pp. 4365 -4376, 2019.

[4] A.S. Khan, P. Dhevagi, P. Chitdeshwari, S. Avudainayagam. "Bio-sorption of cadmium from Aqueous solutions by banana peel powder", Madras Agric Journal, Vol. 105, 24-29, 2018. Doi: https://DOI: 10.29321/MAJ.2018.000096

[5] O.T. Okareh, A.T. Adeolu. "Removal of Lead Ion from Industrial Effluent Using Plantain (Musa paradisiaca) Wastes". British Journal of Applied Science \& Technology, Vol. 8, Pp. 267-276, 2015.

[6] M.R. Chandraiah. "Facile synthesis of Zerovalent Iron magnetic Biochar Composites for $\mathrm{Pb}$ (II) removal from the aqueous medium", Alexandria Engineering Journal, Vol. 55, Pp. 619 - 625, 2016.

[7] F.S. Nworie, F. Nwabue, I.I. Ikelle, A.O. Ogah, N. Elom, N.O. Illochi, E.J. Itumoh, C.E. Oroke. "Activated Plantain Peel Biochar As Adsorbent For Sorption of Zinc(II) Ions: Equilibrium and Kinetics Studies”, Journal of Turkish Chemical Society A, Vol. 5, Pp. 1257 - 1270, 2018.

[8] Y.P. Musa, Z.A. Zurina, A.R. Suraya, M. Faizah, A.S.M. Noor, A. Mohammed. "Eco- Friendly Sustainable Fluorescent Carbon Dots for the Adsorption of Heavy Metal Ions in Aqueous Environment", Nanomaterials, Vol. 10, Pp. 315, 2019. https://doi.org/10.3390/nano10020315

[9] P.D. Pathak, S.A. Mandavgane, B.D. Kulkarni. "Fruit peel waste as a novel low-cost bio adsorbent", Review on Chemical Engineering, Vol. 31, Pp. 361381,2015

[10] D. Moyakhe, F.B, Waanders, M. Le Roux, Q.P. Campbell. "Hydrothermal Preparation of Biochar from Spent Coffee Grounds, and its Application for the Removal of Cadmium from Coal Tailings Leachate", The Journal of the Southern African Institute of Mining and Metallurgy, Vol. 119, Pp. 607-612, 2019. Doi: 10.17159/2411-9717/449/2019.

[11] L. Simeng, G. Chen. “Using hydrogel-biochar composites for enhanced cadmium removal from aqueous media”, Material Sci \& Eng, Vol. 2, Pp. 294-298, 2018. Doi: $10.15406 / \mathrm{mseij} .2018 .02 .00073$

[12] R. Farouq, N.S. Yousef. "Equilibrium and Kinetics Studies of adsorption of Copper (II) Ions on Natural Biosorbent", International Journal of Chemical Engineering and Applications, Vol. 6, Pp. 319 - 324, 2015.

[13] R.P. Mokkapati, V.N. Ratnakaram, J.S. Mokkapati. "Utilization of agro-waste for removal of toxic hexavalent chromium: surface interaction and mass transfer studies”, Int, J. Environ. Sci. Technol, Vol. 10, Pp. 017-037, 2017. Doi: https://DOI:10.1007/s13762-017-1443

[14] N.A. Ebelegi, A.K. Inengite, N. Ayawei, D. Wankasi. "Modeling of Adsorption Isotherms for Methylene Blue Sorption onto Fly Ash Modified with Hydrochloric Acid", Journal of Chemical Science and Technology, Vol. 6, Pp. 1-9, 2017.

[15] P. Sampranpiboon, X. Feng. "Kinetic Models of Chromium (VI) Adsorption onto Carbonized Palm Oil Kernel with Potassium Hydroxide Activation", International Journal of Advances in Chemical Engineering and Biological Sciences (IJACEBS), Vol. 3, Pp. 66-71, 2016. 